\title{
Effects of contrast polarity in paracontrast masking
}

\author{
Hulusi Kafaligönül, Bruno G. Breitmeyer, and Haluk ÖĞMEN \\ University of Houston, Houston, Texas
}

\begin{abstract}
The visibility of a target stimulus can be suppressed (inhibition) or increased (facilitation) during paracontrast masking. Three processes have been proposed to be involved in paracontrast masking: brief inhibition, facilitation, and prolonged inhibition (Breitmeyer et al., 2006). Brief inhibition is observed when the mask precedes the target at short stimulus onset asynchronies (SOAs) ranging from -10 to $-30 \mathrm{msec}$, whereas prolonged inhibition is effective up to very large SOAs of $-450 \mathrm{msec}$. Facilitation, enhancement in target visibility, can be observed at SOA values between -20 and $-110 \mathrm{msec}$. We further investigated these processes by changing target-mask spatial separation and the contrast polarity of the mask. Our results show that (1) facilitation weakens when spatial separation between the target and mask is increased or when they have opposite contrast polarity, and (2) brief inhibition turns into facilitation for the opposite-polarity mask, whereas prolonged inhibition does not change significantly. These results suggest a fast inhibition mechanism realized in the contrast-specific center-surround antagonism of classical receptive fields for brief inhibition and a slower, higher level cortical processing that is indifferent to contrast polarity for prolonged inhibition.
\end{abstract}

Visual masking refers to the reduction in the visibility of a stimulus, called the target, caused by another visual stimulus, called the mask (Breitmeyer \& Öğmen, 2006). Visual masking is a phenomenon deemed worthy of study in its own right but is also a powerful tool for investigating the dynamics of vision, including the interactions between different levels and streams of visual processing (Bachmann, 1994; Breitmeyer \& Öğmen, 2000; Breitmeyer, Öğmen, \& Chen, 2004; Breitmeyer, Öğmen, Ramon, \& Chen, 2005; Öğmen, Breitmeyer, \& Melvin, 2003). When the mask stimulus follows the target stimulus, backward masking prevails, and when it is followed by the target, forward masking prevails. Paracontrast and metacontrast are specific types of forward and backward masking, respectively. In paracontrast and metacontrast masking, target and mask stimuli, although not overlapping in space, are typically close to each other. The plot of target visibility as a function of the stimulus onset asynchrony (SOA) between the target and the mask is called the masking function. By convention, in paracontrast, the SOA is given in negative values, to indicate that the mask occurs before the target.

Although many masking studies have assessed the underlying mechanisms of metacontrast (e.g., Breitmeyer \& Öğmen, 2000, 2006; Francis, 1997; Hermens, Luksys, Gerstner, Herzog, \& Ernst, 2008), only a few studies have addressed paracontrast masking. Recently, Breitmeyer et al. (2006) investigated not only meta- but also paracontrast masking, using tasks requiring observers to judge the surface brightness or else the contours of target stimuli.
Paracontrast masking functions for both tasks are shown in Figure 1 (upper panel). Both brightness match and contour identification tasks tended to yield paracontrast functions with somewhat complicated nonmonotonicities. The brightness judgment task showed enhancement in target visibility at SOAs of about -50 to $-100 \mathrm{msec}$, whereas a weaker suppression effect was evident at longer SOAs. The contour judgment task yielded large suppressive effects, which were maximal at an SOA of $-10 \mathrm{msec}$ and lasted up to SOA values beyond $-350 \mathrm{msec}$. These results have been interpreted in terms of three processes (Breitmeyer et al., 2006): brief inhibition, facilitation, and prolonged inhibition (Figure 1, lower panel). These three processes interact with different magnitudes and produce distinct paracontrast masking functions for surface brightness and contour identification tasks. In other words, a strong (weak) facilitatory effect interacts with very weak (strong) brief and prolonged inhibition processes for surface brightness (contour identification) task and yields the net paracontrast masking function, as shown in Figure 1 (upper panel).

Brief inhibition is observed at short SOA values (between 0 and $-30 \mathrm{msec}$ ) and may be caused by centersurround inhibition of the classical receptive fields. It has been emphasized that the inhibitory surround activation of classical receptive fields is slower than activation of the center region by 10-30 msec (Benardete \& Kaplan, 1997; Maffei, Cervetto, \& Fiorentini, 1970; Poggio, Baker, Lamarre, \& Sanseverino, 1969; Singer \& Creutzfeldt, 1970). As a result of this asynchrony, the surrounding mask has

H. Kafaligönül, hulusi.kafaligonul@gmail.com 


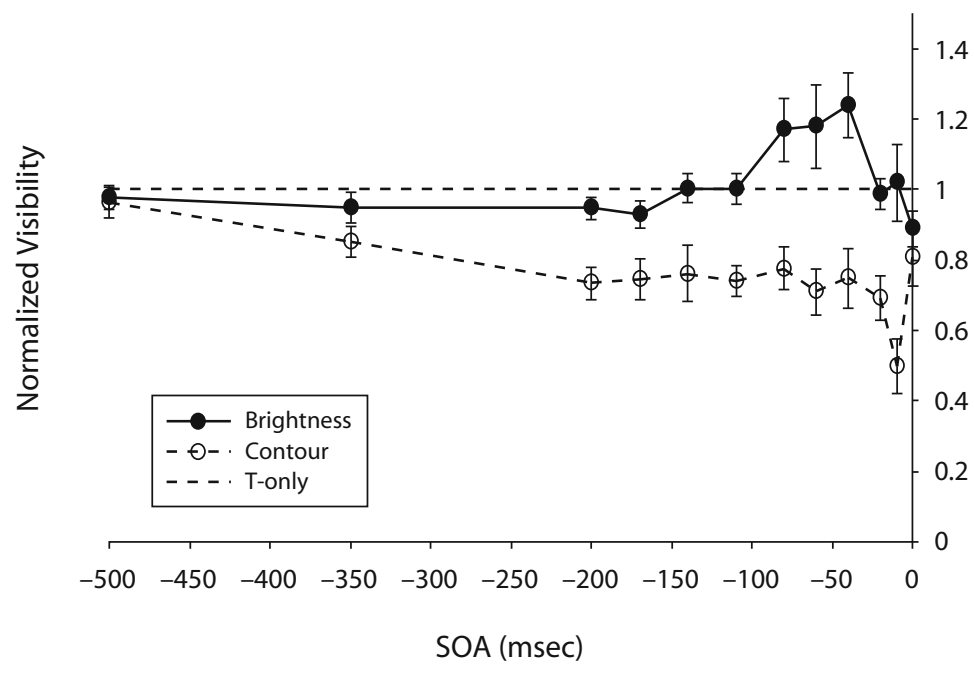

Paracontrast Mechanisms

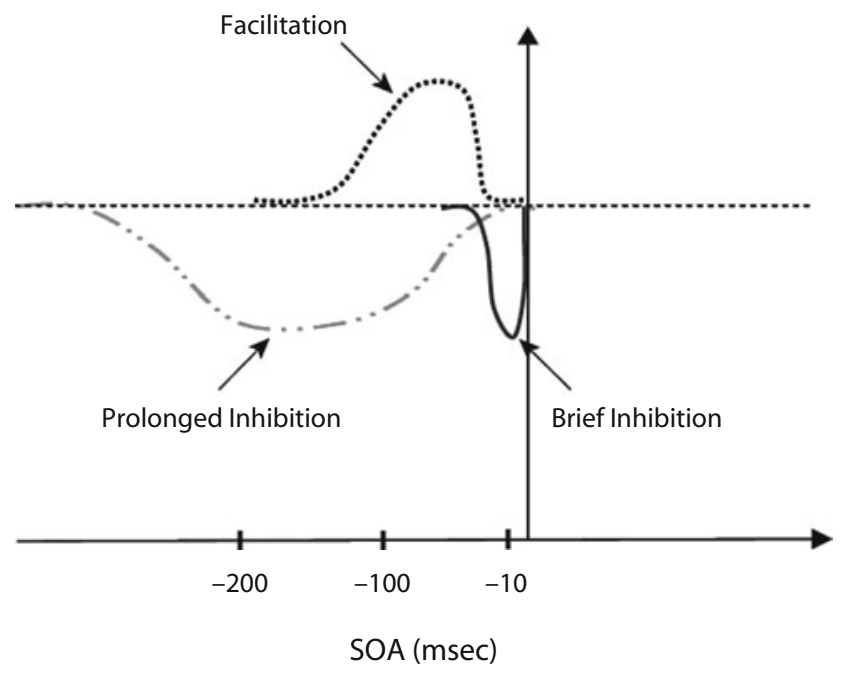

Figure 1. Upper panel: Averaged data across three mask-to-target (M/T) contrast ratios $(0,0.5,1.0)$ for surface brightness and contour identification tasks. The visibility values are normalized with respect to the target-only (T-only) condition, and the error bars represent $\pm S E M$. Lower panel: Schematic diagram of the three processes proposed to underlie paracontrast masking: brief inhibition, facilitation, and prolonged inhibition. From "Meta- and Paracontrast Reveal Differences Between Contour- and Brightness-Processing Mechanisms," by B. G. Breitmeyer et al., 2006, Vision Research, 46, p. 2651. Copyright 2006 by Elsevier. Reprinted with permission.

to precede the target by SOAs of 10-30 msec to obtain optimal suppression of target-induced excitatory activity. Paracontrast results (Breitmeyer et al., 2006; Öğmen et al., 2003) also indicate an additional inhibitory effect lasting up to $-450 \mathrm{msec}$. Similar prolonged (temporal offsets larger than $200 \mathrm{msec}$ ) inhibition was shown in the visual cortex of the cat (Berman, Douglas, Martin, \& Whitteridge, 1991; Nelson, 1991) and both (prolonged and brief) inhibition mechanisms were used by several modeling studies of the visual cortex (Ursino \& La Cara, 2004a, 2004b).
The facilitatory effect of a preceding mask on the visibility of a target can be observed with different types of stimuli (Bachmann, 1988, 1994, 1997; Foley \& Chen, 1999; Georgeson, 1988; Polat \& Sagi, 1993, 2006; Tanaka \& Sagi, 1998; Xing \& Heeger, 2000). Bachmann (1988, 1994, 1997) emphasized the interaction between the signals carried out by nonspecific (e.g., subcortical brain stem and midbrain) pathways and specific (i.e., the retino-geniculo-cortical) afferent pathways. He further suggested that the slower nonspecific subcortical activation of a preceding mask modulates the faster specific 
cortical activity generated by the target stimulus and thus enhances the perceived target visibility. Recently, in several studies by Polat (Polat \& Sagi, 2006; Polat, Sterkin, \& Yehezkel, 2007), forward and backward masking were examined by using oriented Gabors as target and mask stimuli. In these studies, both facilitatory and inhibitory effects of the mask stimulus were shown. Furthermore, the facilitatory effect was observed mainly when the target and mask did not overlap in space significantly and the mask appeared before the target in time. They implicitly generalized these findings to forward and backward masking and suggested that the facilitatory effect of the mask was the result of lateral long-range interactions and perceptual grouping mechanisms in the visual system (primarily in V1; Polat \& Sagi, 1993, 2006; Polat et al., 2007). They also proposed that facilitatory signals propagate in space with a relatively slow velocity $\left(3^{\circ} / \mathrm{sec}\right)$ and reach distant locations after some delay (Polat \& Sagi, 2006; Tanaka \& Sagi, 1998).

In this study, we investigated the underlying mechanisms of the facilitation and tested the hypothesis based on lateral long-range connections by changing targetmask (T-M) separation in our paracontrast masking experiment. We used a disk and a surrounding ring as the target and mask, since this stimulus configuration is traditionally used in masking studies and allows us to compare the results more directly with those of our previous study (Breitmeyer et al., 2006). Our aim was also to characterize further the three processes proposed for paracontrast masking and extend our previous distinctions between brightness and contour processing by observing their contrast polarity dependence. Analyzing contrast polarity effects has been fruitful in assessing the potential mechanisms for masking and metacontrast (e.g., Becker \& Anstis, 2004; Breitmeyer, 1978; Breitmeyer, Tapia, Kafaligönül, \& Öğmen, 2008; Otto, Öğmen, \& Herzog, in press; Sayim, Westheimer, \& Herzog, 2008), and we took a similar approach for paracontrast.

\section{EXPERIMENT 1}

\section{Rationale: What Is the Source of the Facilitatory Mechanism?}

In this experiment, we investigated the temporal dynamics and the underlying mechanisms of the facilitation observed in paracontrast masking. As was mentioned above, several studies have pointed out the contribution of lateral excitatory connections in the cortex to the visibility of a target stimulus. These excitatory signals propagate to the location of the target with a time delay and increase its visibility. An increase in T-M separation should result in an increase in this time delay. If these connections are the main cause of the facilitation observed in paracontrast masking, the SOA value at which maximum facilitation occurs should shift to more negative values (longer SOAs) as a result of an increase in the T-M separation (Figure 2). In order to test this hypothesis, we followed mainly the methods reported in our previous studies (Breitmeyer et al., 2006; Öğmen et al., 2003) and systematically changed $\mathrm{T}-\mathrm{M}$ separation.

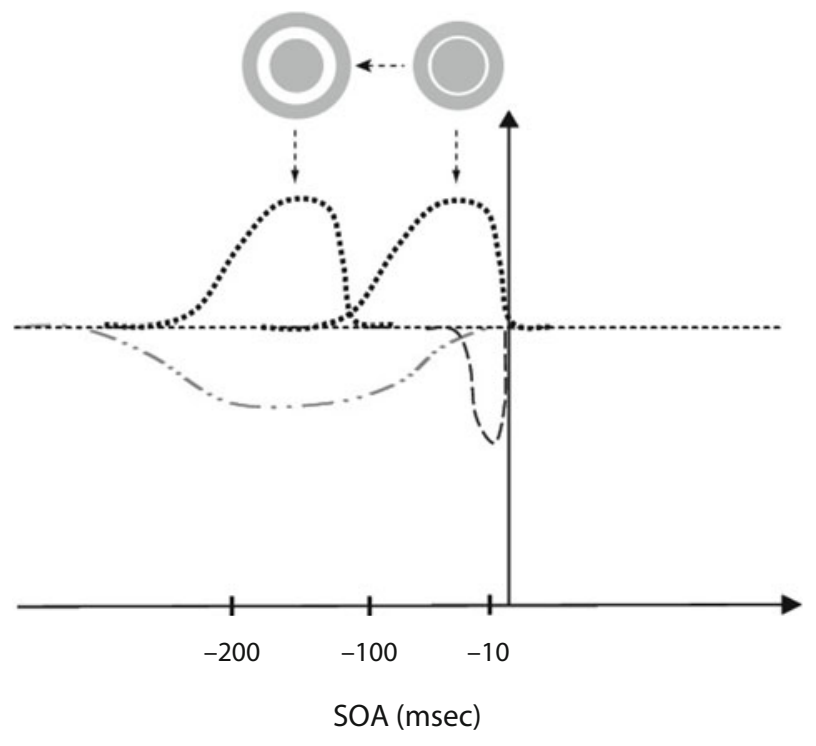

Figure 2. Illustration of the prediction based on lateral excitatory connections in the visual cortex. According to the hypothesis based on lateral excitatory connections, the peak facilitation should shift to more negative stimulus onset asynchrony (SOA) values as target-mask separation increases.

\section{Method}

Apparatus. The experiment was performed in a dark room. Visual stimuli were generated via a Cambridge Research Systems (CRS) VSG2/3 board working in a PC computer. This board was programmed by using its driver library. The stimuli were displayed on a NANAO F2-21 monitor, and its frame rate was $100 \mathrm{~Hz}$. The display was gamma corrected, and luminance calibrations were periodically checked by a Minolta LS-110 luminance meter and a CRS ColorCAL colorimeter. A head- and chinrest stabilized fixation. The distance between the display and the observer was set to $98 \mathrm{~cm}$. The responses of the observer for each trial were recorded with a joystick connected to the computer. The same apparatus was used in all the experiments.

Stimuli and Procedure. The upper panel in Figure 3 indicates a typical stimulus configuration used in the experiment. The fixation mark consisted of a small $\left(0.4^{\circ} \times 0.4^{\circ}\right)$ dark $\left(0.5 \mathrm{~cd} / \mathrm{m}^{2}\right)$ cross in the center of the screen. The target was a disk of $0.85^{\circ}$ diameter and centered $1.6^{\circ}$ to the right of the fixation cross. The mask ring had a width of $0.4^{\circ}$ and it surrounded the target disk. A comparison disk of $0.85^{\circ}$ diameter was centered $1.6^{\circ}$ to the left of the fixation cross. Background luminance was $10 \mathrm{~cd} / \mathrm{m}^{2}$, and the target and mask had a luminance of $30 \mathrm{~cd} / \mathrm{m}^{2}$. The target, mask, and comparison stimuli all had the same duration of $10 \mathrm{msec}$. We used three T-M separation (i.e., distance between target outer edge and mask inner edge) values: $0^{\circ}, 0.15^{\circ}$, and $0.30^{\circ}$. We kept the width of the mask ring constant for these different $\mathrm{T}-\mathrm{M}$ separations. For the $0^{\circ} \mathrm{T}-\mathrm{M}$ separation condition, the mask had inner and outer diameters of $0.85^{\circ}$ and $1.25^{\circ}$, respectively. These conditions were run in separate sessions, and the order of these sessions was randomized across observers. Each condition had 10 SOA values $(0,-10,-20,-40,-60,-80$, $-110,-140,-170$, and $-200 \mathrm{msec}$ ), in addition to a no-mask (target-only) case. The no-mask (target-only) case was used in order to measure baseline match values and to normalize data with respect to these baseline values. The order of these 11 cases was randomized during each session.

We employed a direct measure of perceived brightness. The fixedluminance target and a variable-luminance comparison stimulus were presented simultaneously. After each presentation (including the preceding mask), the observers were required to indicate, by 


\section{Same-Contrast-Polarity Condition}

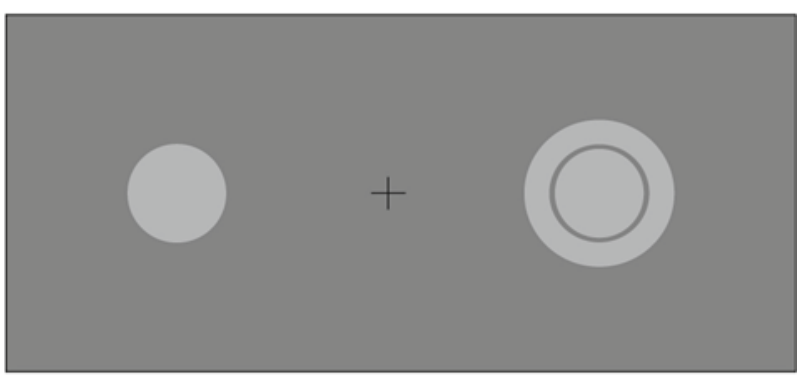

Opposite-Contrast-Polarity Condition

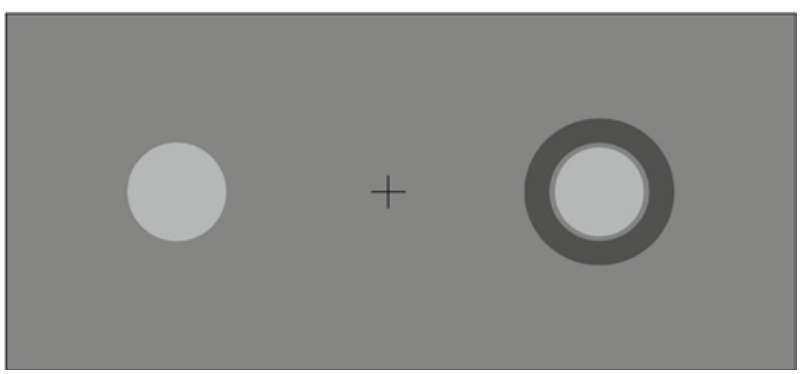

Figure 3. Stimulus configuration used in Experiments 1, 2, and 4 . Although there is a gap between the target and mask in the figure for clarity, the target-mask separation was zero for samecontrast-polarity (upper panel) and opposite-contrast-polarity (lower panel) conditions in Experiments 2 and 4. Plus signs indicate the fixation cross. The comparison and target disks were shown $1.6^{\circ}$ to the left and right of the fixation cross, respectively. The mask annulus surrounded the target disk, and it was presented at different stimulus onset asynchrony values.

pressing one of two keys, which of the two, target or comparison, appeared brighter. An up-down staircase procedure was used to determine the point of subjective equality (PSE), defined as the average of the last six reversals (at which the luminance changes were set to the 12-bit luminance resolution) out of a total of nine reversals. For offline data analysis, all PSE values were expressed in terms of Michelson contrast. Two naive observers and 2 of the authors (B.G.B. and H.K.) participated in this experiment. The observers in all our experiments had normal or corrected-to-normal vision and were given practice sessions before the start of formal data collection so as to stabilize their performance.

\section{Results and Discussion}

The results are based on normalized target visibilities. For each session, perceived target brightness (the PSE Michelson contrast value) at each SOA was normalized by dividing it by the target's perceived brightness obtained in the baseline, no-mask condition. These normalized visibilities averaged across 4 observers are shown at each $\mathrm{T}-\mathrm{M}$ separation in Figure 4. When there is no T-M separation, we observe a facilitation effect between SOAs of 0 and $-110 \mathrm{msec}$, and the normalized visibility function peaks around $-40 \mathrm{msec}$. These SOA values are consistent with our previous results in Breitmeyer et al. (2006). As $\mathrm{T}-\mathrm{M}$ separation increases, the facilitation effect starts decreasing. However, the overall results do not show a systematic shift of the peak facilitation to more negative SOA values. If anything, it tends to shift to less negative SOA values. Another observation is that $\mathrm{T}-\mathrm{M}$ separation does not affect the suppression effect observed between SOA values of -110 and $-200 \mathrm{msec}$.

In support of the aforementioned observations, a statistical analysis of the data shows that the effects of SOA were significant $[F(9,27)=9.001, p=.030, \varepsilon=0.167]$. The overall effect of $\mathrm{T}-\mathrm{M}$ separation and its interaction with SOA were not significant $[F(2,6)=2.145, p=.198$, and $F(18,54)=1.446, p=.303, \varepsilon=0.128$, respectively]. However, the T-M separation was found to be significant $[F(2,6)=8.625, p=.017]$ in the SOA range (values between -20 and $-110 \mathrm{msec}$ ) where facilitation in the target's visibility was observed.

\section{EXPERIMENT 2}

\section{Rationale: Effect of Contrast Polarity on Paracontrast Masking in Surface Brightness Judgments}

In metacontrast, the effect of stimulus contrast polarity has been used to infer potential mechanisms underlying the reduction of the target's visibility (e.g., Becker \& Anstis, 2004; Breitmeyer, 1978; Breitmeyer et al., 2008). In this and the following experiments, our goal was to take a similar approach by investigating the contrast polarity dependence of brief inhibition, of facilitation, and of prolonged inhibition mechanisms. Previous studies with sinusoidal gratings and Gabor stimuli showed that facilitation is dependent on the phase difference between target and mask stimuli (Georgeson, 1988; Tanaka \& Sagi, 1998). As was mentioned in the introduction, the facilitation is observed mainly in the paracontrast masking function of the surface brightness judgment task. Therefore, we used a surface brightness judgment task in this experiment to observe the effect of contrast polarity on the facilitation mechanism.

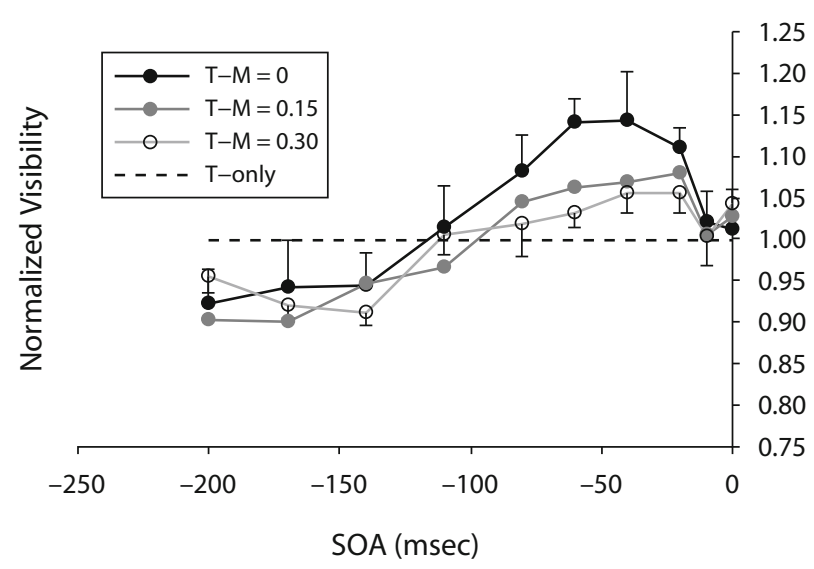

Figure 4. Results of Experiment 1. The averaged normalized visibility of the target as a function of stimulus onset asynchrony (SOA) for 4 observers. Results for different target-mask (T-M) separations are with respect to the target-only (T-only) baseline condition, which is normalized to a value of 1 (dotted line). Error bars correspond to $1 S E M$. The error bars for the $\mathrm{T}-\mathrm{M}=0.15^{\circ}$ condition were similar to error bars in the $\mathrm{T}-\mathrm{M}=0.30^{\circ}$ condition and are not included in the figure, to avoid clutter. 


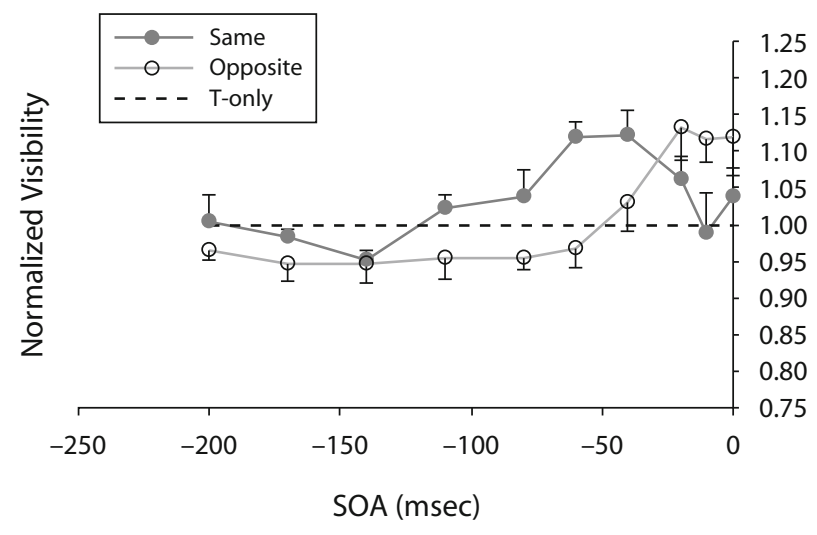

Figure 5. Results of Experiment 2. The averaged normalized visibility of the target as a function of stimulus onset asynchrony (SOA) for 4 observers. Results for different polarity conditions are with respect to a target-only (T-only) baseline condition, which is normalized to a value of 1 (dotted line). Error bars correspond to $1 S E M$.

\section{Method}

Stimuli and Procedure. The experiment consisted of two conditions, depicted in Figure 3, defined by same and opposite contrast polarity of the target and mask, and each condition was run in separate sessions. Background luminance was $10 \mathrm{~cd} / \mathrm{m}^{2}$. In the same-contrast-polarity condition, the target and mask had a luminance (above background) of $30 \mathrm{~cd} / \mathrm{m}^{2}$. The mask had the luminance (below background) of $3.3 \mathrm{~cd} / \mathrm{m}^{2}$ in the opposite-polarity condition, yielding the same absolute value of the two masks' Michelson contrasts. T-M separation was zero, and we used the same stimulus parameters and SOA values as those in the first experiment. The perceived visibility of the target disk was measured as before, and the average normalized visibility values of 4 observers ( 2 naive and 2 authors) were plotted for different SOA values.

\section{Results and Discussion}

The results are shown in Figure 5. The effect of SOA on paracontrast masking functions was significant $[F(9,27)=6.946, p=.026, \varepsilon=0.227]$. In the same-contrast-polarity condition, we observed, as in Experiment 1, a facilitation effect between SOAs of 0 and $-110 \mathrm{msec}$, and the normalized visibility function peaked around $-40 \mathrm{msec}$. In the opposite-contrastpolarity condition, the facilitation peaked at $\mathrm{SOA}=$ $-20 \mathrm{msec}$ and then decreased and turned into a relatively weak suppression for more negative SOA values. Although the overall main effect of mask polarity was not significant $[F(1,3)=3.038, p=.180]$, for the seven SOA values between 0 and $-110 \mathrm{msec}$ where facilitation is mainly observed, the $\mathrm{SOA} \times$ mask polarity interaction was significant $[F(6,18)=6.620, p=.039, \varepsilon=0.289]$, indicating that the morphology of facilitation depended on the contrast polarity of the stimulus.

The contrast-polarity-dependent change in the morphology of facilitation as a function of SOA can be interpreted in terms of the three putative mechanisms suggested in our previous study (Breitmeyer et al., 2006; see Figure 1). Our model suggests that the morphology of the paracontrast function is determined by a combination of a prolonged inhibition, brief inhibition, and facilita- tion (Breitmeyer et al., 2006). According to our results shown in Figure 5, when the target and mask have the same contrast polarity, there is no net brief inhibition at short SOA values. In other words, the data points at these SOA values are not significantly below baseline. However, the visibility consistently drops around $-10 \mathrm{msec}$, relative to the neighboring SOA values. The dip around $-10 \mathrm{msec}$ could thus be the effect of strong facilitatory and relatively weaker brief inhibitory processes jointly determining a net paracontrast masking function, as depicted schematically in the upper panel of Figure 6. We therefore interpret the visibility drop at $-10 \mathrm{msec}$ as a consequence of the proposed brief inhibition, even if normalized target visibility values are not below one. Let us highlight that even though the interpretation of the visibility drop as inhibition is indirect in this context, the brief inhibition as a drop below baseline can be seen

\section{Same-Contrast Polarity}

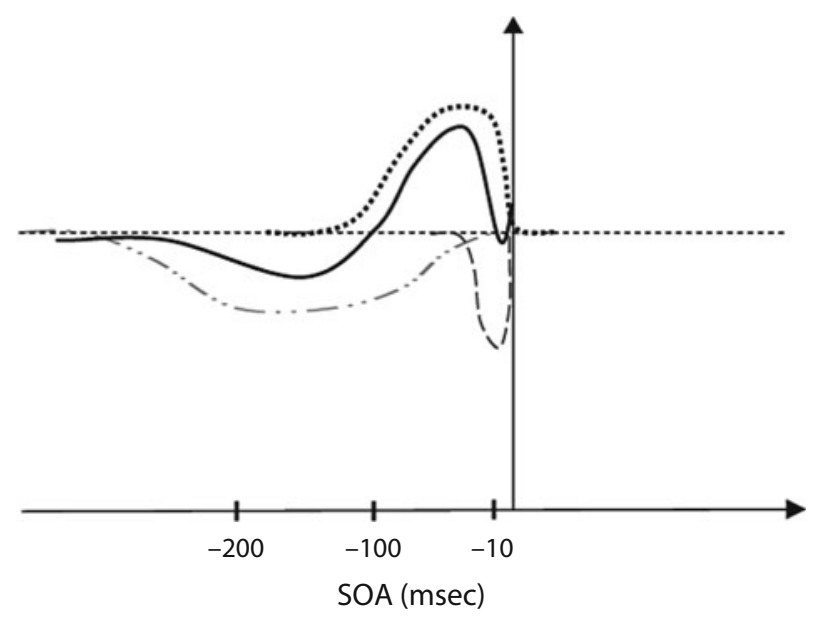

Opposite-Contrast Polarity

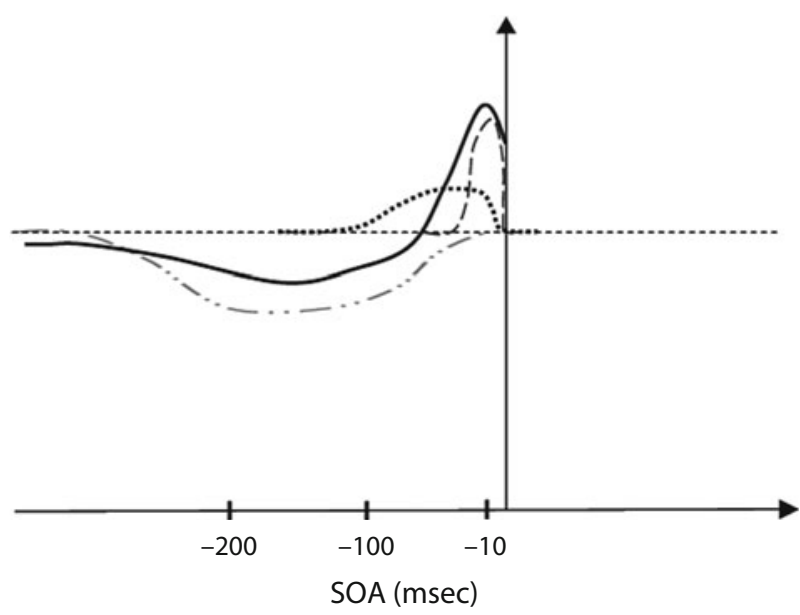

Figure 6. Illustration of how the proposed mechanisms explain the results of Experiment 2. Same- and opposite-polarity conditions are drawn separately. The continuous line represents the net paracontrast masking function as a result of these three mechanisms. SOA, stimulus onset asynchrony. 


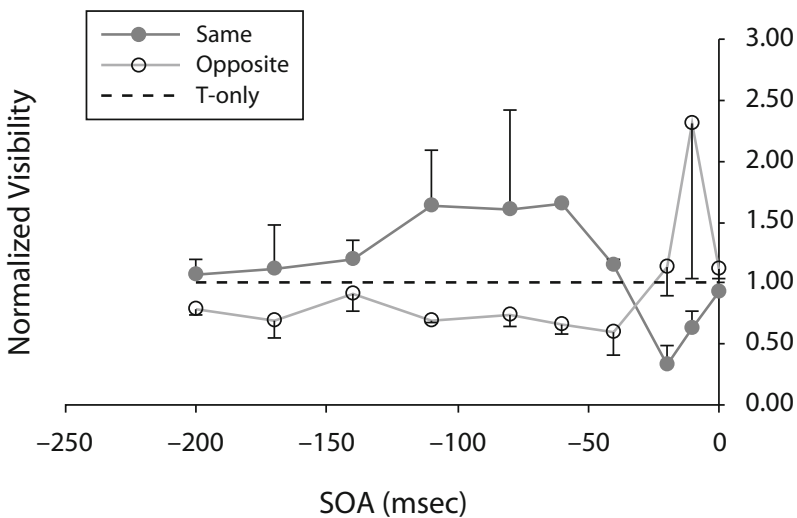

Figure 7. Results of a preliminary experiment that was identical to Experiment 2, with the exception of the following parametric changes. The background and the target luminance values were 15 and $10 \mathrm{~cd} / \mathrm{m}^{2}$, respectively. The mask luminance was 10 (22.5) $\mathrm{cd} / \mathrm{m}^{2}$ in the same- (opposite-) contrast-polarity condition. These preliminary data were collected from 1 observer (H.K.) and only two runs. Error bars indicate $S E M$ obtained from these two runs. SOA, stimulus onset asynchrony.

in other experiments in which different parameter values have been used. For example, the inspection of data in the upper panel of Figure 3 in Breitmeyer et al. (2006) shows a clear drop below baseline when the mask-target contrast ratio equals 0.5 . Figure 7 shows unpublished data from our laboratory obtained with background, target, and mask luminance values different from those used in this study. Again, a clear drop below baseline can be seen when the target and mask have the same contrast polarity.

Taken together, these results support the existence of a brief inhibitory process that interacts with a facilitatory process and a prolonged inhibitory process (with different weights according to stimulus parameters) to determine the net morphology of the paracontrast function. This interpretation can be further tested by the following prediction: If, as is assumed, the brief inhibition results from surround inhibition within a classical on-center offsurround receptive field, inverting the polarity of the mask should lead to facilitation, instead of inhibition, because, as is shown in Figure 8, inverting the polarity of the mask reduces the energy delivered to the inhibitory surround of the receptive field, thereby reducing the inhibition with respect to the baseline target-only condition. In turn, changing the brief inhibition to a brief facilitation should modify the morphology of the paracontrast function according to the depiction shown in the bottom panel of Figure 6. Thus, unlike in the same-polarity condition, where paracontrast function exhibits a drop for SOAs near zero, in the opposite-contrast-polarity condition, the facilitation should be observed for all SOAs near zero. The data in Figure 5 support this interpretation.

In the following experiment, we applied the contrast polarity test to the contour judgment task to obtain insight into the mechanisms involved in the computation of contours.

\section{EXPERIMENT 3}

\section{Rationale: Effect of Contrast Polarity on Paracontrast Masking, Contour Discrimination Judgments}

We repeated Experiment 2, using a contour identification task instead of surface brightness. According to several studies, the processing of illusory contours and figure-ground segregation are independent of the contrast polarity of the inducers (Dresp \& Bonnet, 1995; Peterhans \& von der Heydt, 1989; Zhou, Friedman, \& von der Heydt, 2000). Moreover, contour processing has been proposed to be insensitive to contrast polarity by several modeling studies (Grossberg, 1994, 2003; Yen \& Finkel, 1998). On the basis of these findings, in the case of contour judgments, we would expect the paracontrast function to be relatively insensitive to changes in contrast polarity, unlike the paracontrast functions obtained by brightness judgments.

\section{Method}

Stimuli and Procedure. The parameters of the stimuli were the same as the parameters in Experiment 2. Percent correct in a contour identification task was measured, instead of perceived brightness. The target could consist of a complete disk, a disk with a $0.38^{\circ}$ wide upper contour deletion or a disk with the lower contour deletion of the same size (Figure 9). T-M separation was zero. The target, preceded by the surrounding mask, could be shown $1.6^{\circ}$ to the left or right of the fixation cross. Each experimental session had $11 \mathrm{SOA}$ values $(0,-10,-20,-40,-60,-80,-110,-140,-170,-200$, and $-350 \mathrm{msec}$ ) and a no-mask (target-only) case, and the order of these 12 cases was randomized. The target-only condition was used to obtain a baseline performance during the experiment. At each $\mathrm{SOA}$, the location of the T-M sequence was randomized across 30 trials, with half of the trials devoted to the left location, the remaining half to the right location. Of the 30 trials, 10 were devoted to each of the three possible target contours. The order of target contours was randomized across the 30 trials. After each trial, the observers were required to indicate, by pressing one of three keys, which of the three targets had been presented. Each condition (same and opposite polarity) was run in separate sessions, and the order of these sessions was randomized across observers. Two naive observers and 2 of the authors (B.G.B. and H.K.) participated in this experiment.

\section{Results and Discussion}

In order to compare the contour identification results with surface brightness measures, the performance values in the contour identification task were also normalized according to the baseline (T-only) condition, as in Experiments 1 and 2, for surface brightness. This normalization also allowed us to eliminate the potential effects of position uncertainty (which exists in both the paracontrast and target-only conditions) and to observe the main masking effect in the contour identification task.

The average normalized visibility in the contour identification task is shown in Figure 10 (upper panel). Both contrast polarity conditions yielded paracontrast functions with significant dependence on SOA values $[F(10,30)=$ $10.405, p=.012, \varepsilon=0.194]$. In agreement with the results of Breitmeyer et al. (2006), the contour identification task indicated a dominant suppression effect lasting up to longer SOA values of $-350 \mathrm{msec}$. The overall ef- 


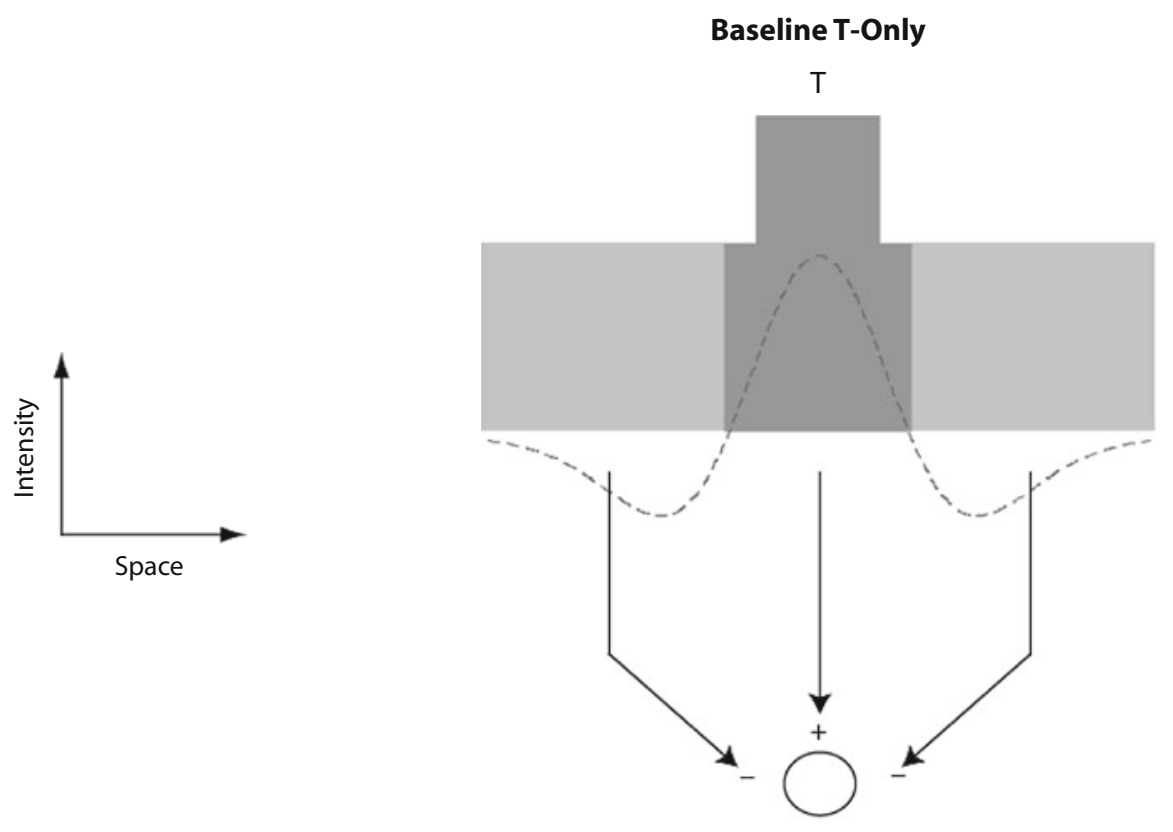

T-M Same Polarity

M

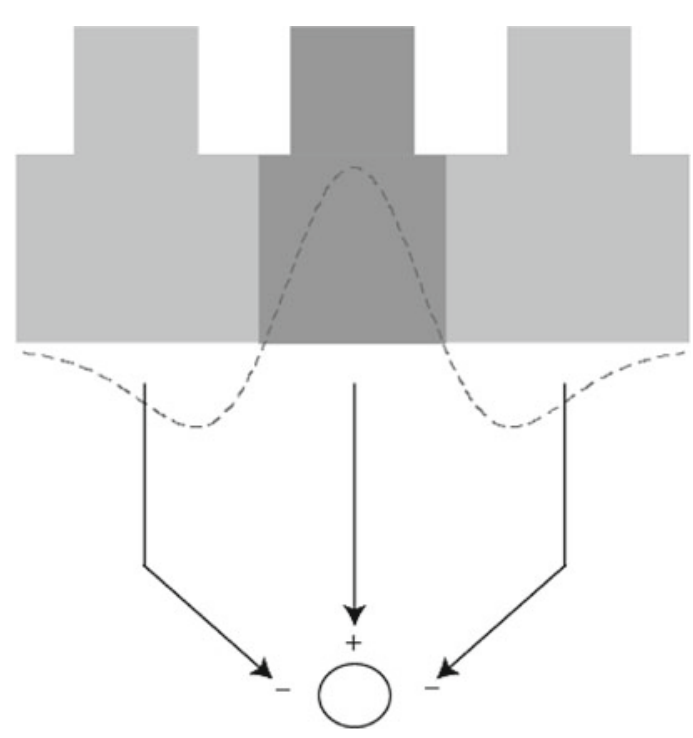

Excitation Same

More Input on Inhibitory Zone

Net Signal < Baseline
T-M Opposite Polarity

$\mathrm{T}$

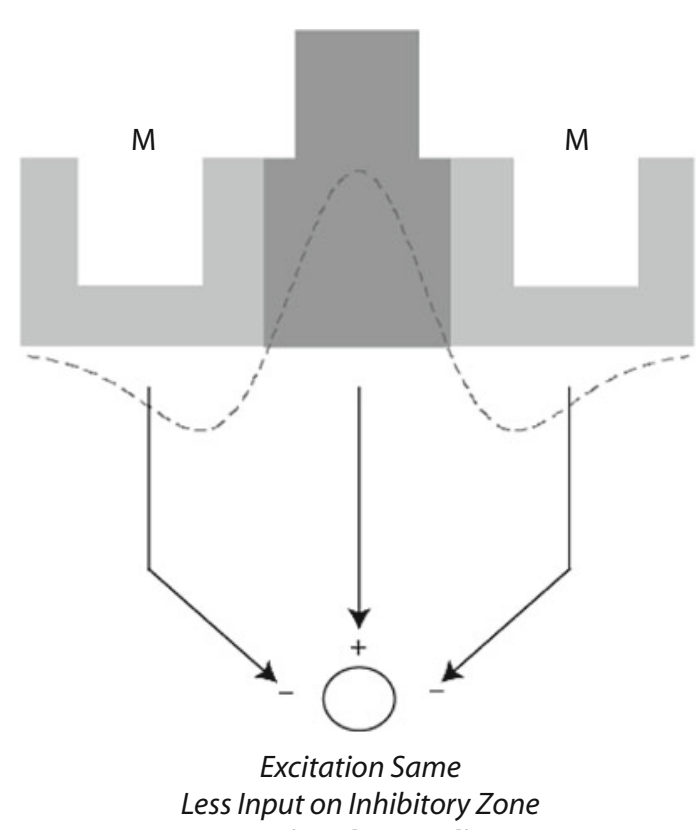

Net Signal > Baseline

Figure 8. Illustration of how brief inhibition turns into facilitation as a result of change in the contrast polarity of a surrounding mask. It has been proposed that the brief inhibition observed at short negative stimulus onset asynchrony values is caused by the surround inhibition of retinal receptive fields. The excitatory (central) zones are shown by the darker regions in the figure. The target and mask luminance levels are shown by increments above the background. The upper drawing represents the target-only (T-only, baseline) condition. The same- and opposite-contrast-polarity conditions are in the lower left and right panels, respectively. In the same-polarity condition, the target visibility is lower than that in the T-only condition, due to the additional luminance input falling on the inhibitory zone of the cell. However, there is less luminance input falling on the inhibitory zone when the target and the mask have opposite-contrast polarity. Therefore, we expect the target visibility to be higher in the opposite-contrast-polarity condition than in the baseline condition. T, target; $M$, mask. 


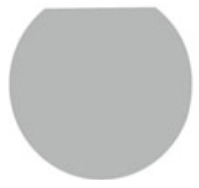

Upper Contour Deletion

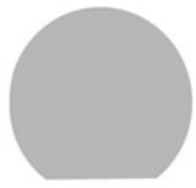

Lower Contour Deletion

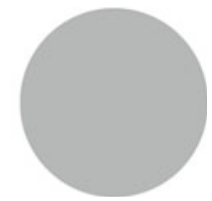

Complete Disk
Figure 9. Three possible target stimuli used in Experiment 3: upper contour deletion, lower contour deletion, and complete.

fect of polarity was not significant $[F(1,3)=0.571, p=$ $.505]$, whereas its interaction with SOA was significant $[F(10,30)=6.412, p=.035, \varepsilon=0.192]$. Although there was a significant change between the two polarity conditions at the SOA values of $0,-10$, and $-20 \mathrm{msec}$, the overall trend of the paracontrast masking function was not affected by a change in the contrast polarity of the mask. When we excluded the three SOA values $(0,-10,-20)$, the polarity $[F(1,3)=2.837, p=.191]$ and its interaction with SOA $[F(7,21)=0.578, p=.568, \varepsilon=0.242]$ were insignificant. Since the T-M separation was zero, the target and mask were perceived as a single circle at $\mathrm{SOA}=0 \mathrm{msec}$ in the same-contrast-polarity condition, and therefore, the presence or absence of a deleted part of the target component could be easily detected by the observers. The same-contrast-polarity condition appears to have had stronger inhibition than the opposite condition, as can be seen by the difference between masking functions for SOA values ranging from -110 to $-350 \mathrm{msec}$. However, this difference was found to be insignificant $[F(1,3)=4.429, p=.126]$. In agreement with the previous studies cited in the Rationale: Effect of Contrast Polarity on Paracontrast Masking, Contour Discrimination Judgments section, the results confirm that contour processing is largely insensitive to the sign of the contrast.

\section{EXPERIMENT 4}

\section{Rationale: Comparison With Simultaneous Brightness Contrast}

The results of Experiment 2 show that at short SOA values $(0,-10$, and $-20 \mathrm{msec})$, the enhancement in target visibility for the opposite-contrast-polarity condition is much larger than that for the same-contrast-polarity condition (Figure 5). Perceived brightness of a static object is affected by the presence of an adjacent surrounding area. For instance, surface brightness of a square appears brighter as the surrounding area becomes darker. This wellknown illusion is called simultaneous brightness contrast (Heinemann, 1955). The main difference between the $\mathrm{SOA}=0 \mathrm{msec}$ condition in our experiment and the classical simultaneous brightness contrast effect is the duration of the visual stimuli. In order to investigate whether the enhancement we found in the opposite-polarity condition and the simultaneous brightness contrast phenomenon are related to each other and share common mechanisms, we varied target and mask durations from the $10 \mathrm{msec}$ used in Experiment 2 to $210 \mathrm{msec}$, which represents simultaneous brightness contrast, and assessed whether the perceived brightness enhancement was quantitatively similar in paracontrast (short exposure duration) and simultaneous brightness contrast (long exposure duration) conditions.

In prior related work, Alpern (1963) explored the effect of exposure duration on the perceived brightness of a central bar (test patch) in the presence of two neighboring bars (inducing patches). The test and inducing patches had the same dimensions $\left(0.05^{\circ} \times 2.5^{\circ}\right)$ and the edge-to-edge separation between the test and the inducing patches was $0.70^{\circ}$. A comparison bar $\left(0.05^{\circ} \times 0.25^{\circ}\right)$ with no surrounding patches was also used, and the observers varied the luminance of the test patch until its perceived brightness matched that of the comparison bar, which had a fixed luminance of $6.57 \mathrm{Ft}-\mathrm{L}$. All of the stimuli were shown simultaneously for different exposure durations. The luminance values of the inducing patches were varied from $794 \mathrm{Ft}-\mathrm{L}$ to $79,432 \mathrm{Ft}-\mathrm{L}$. At the highest inducing bar luminance value, the matching luminance value of the central bar reached its peak at an exposure duration of about $20 \mathrm{msec}$ and, thereafter, decayed to a lower steady state value. As the luminance values of the inducing bars decreased, (1) overall, the matching luminance of the central bar decreased for all durations; (2) the peak matching lu-

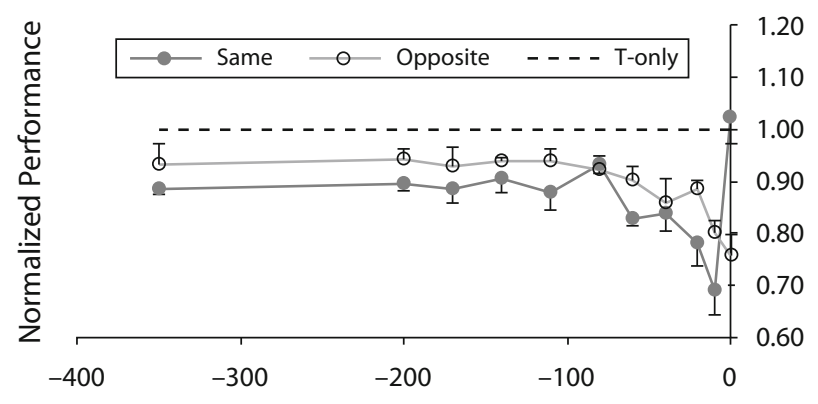

SOA (msec)

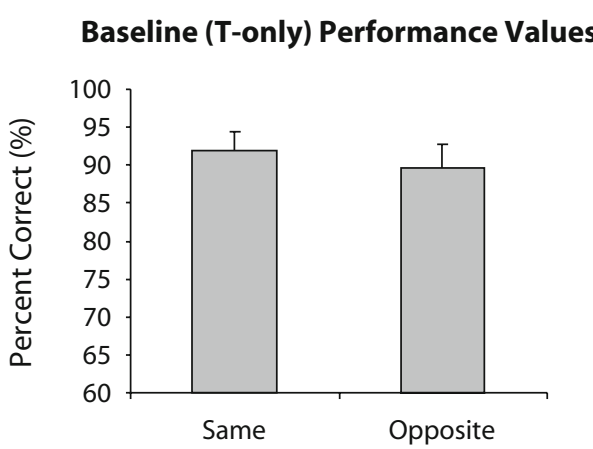

Figure 10. Results of Experiment 3. Upper panel: The normalized performance results are averaged across 4 observers for the contour identification task. Results for different-polarity conditions are with respect to the target-only (T-only) baseline condition, which is normalized to a value of 1 (dotted line). Error bars correspond to $1 S E M$. Lower panel: The averaged baseline (T-only) performance values across observers for different contrast polarity conditions. The baseline values for the same- and opposite-polarity conditions were not significantly different from each other [paired-samples $t$ test: $t(3)=1.155, p=.332$ ]. 
minance value occurred at longer exposure durations; and (3) the difference between peak and plateau decreased, and this yielded more monotonic curves (Alpern, 1963).

In the present experiment, we chose T-M SOAs of 0 , -10 , and $-20 \mathrm{msec}$, corresponding to the brightness enhancement region in the opposite-contrast-polarity condition for paracontrast (Figure 5), and varied systematically the exposure duration from the value used in the paracontrast experiment $(10 \mathrm{msec})$ to a long enough value suitable for simultaneous brightness induction $(210 \mathrm{msec})$.

\section{Method}

Stimuli and Procedure. The parameters were the same as those in the opposite-contrast-polarity condition in Experiment 2. The target and mask had the same exposure durations, which could assume one of the following six values: 10, 50, 90, 130, 170, or $210 \mathrm{msec}$. The comparison disk had a constant exposure duration of $50 \mathrm{msec}$, so that the measures obtained for different stimulus durations were all relative to an identical reference duration. The exposure duration of the comparison disk was chosen to be $50 \mathrm{msec}$ (instead of $10 \mathrm{msec}$, as in the previous experiments) to avoid saturation due to the maximum luminance of the monitor. The SOA value was set at $0,-10$, or $-20 \mathrm{msec}$ during an experimental session. Each experimental session also included baseline target-only (no-mask) trials for these exposure durations. At each SOA, the perceived brightness of the target was measured for the 12 combinations of six exposure durations and two target presentations (with or without mask) by using the same procedures as those in Experiment 1. The order of these conditions was randomized in an experimental session, and the order of each session was randomly interleaved across observers. Two naive observers and one of the authors (H.K.) participated in this experiment.

\section{Results and Discussion}

Figure 11 shows the normalized visibility values of the target for different exposure durations. Since the perceived brightness of the target for no-mask (target-only) condi-
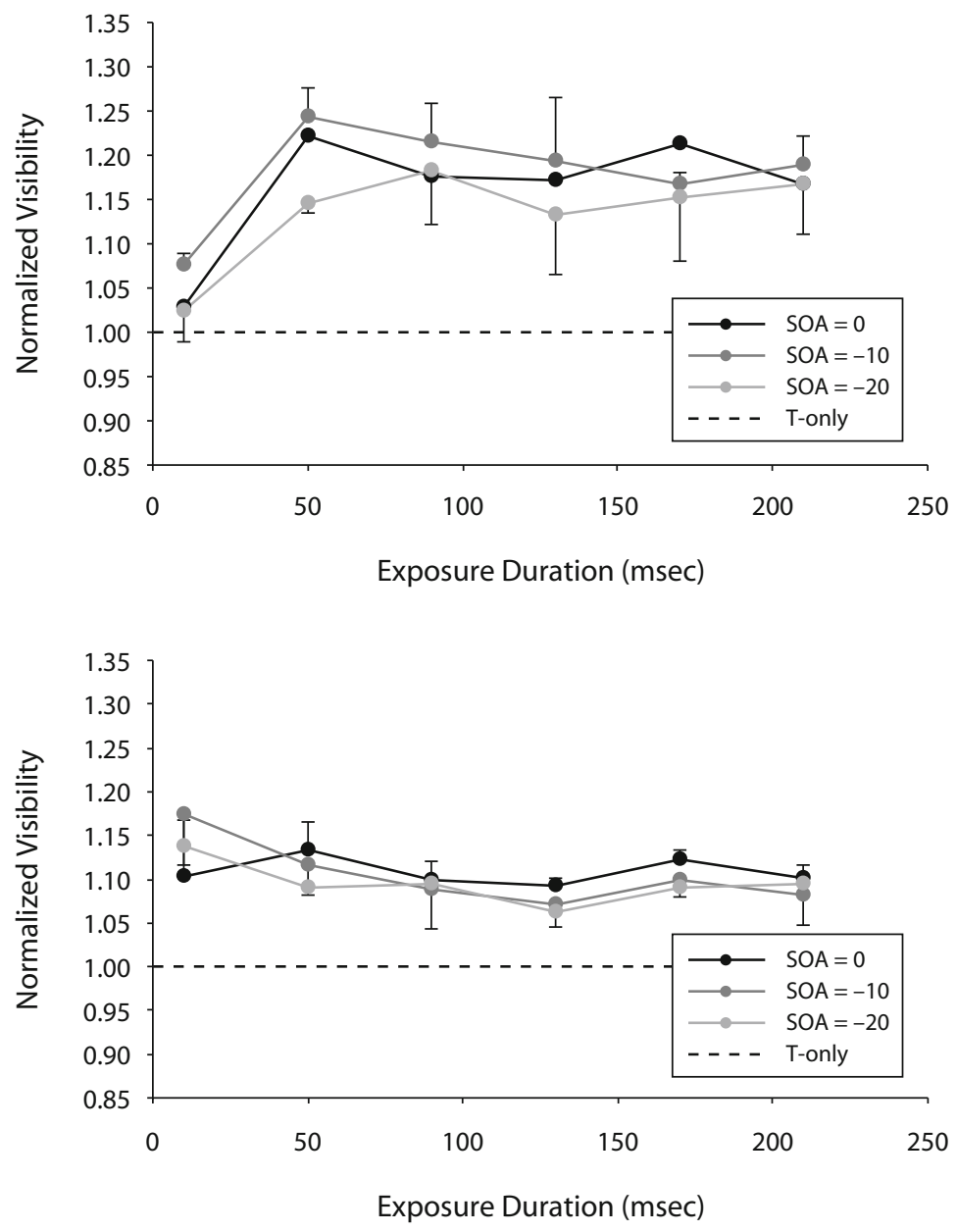

Figure 11. Results of Experiment 4. The averaged normalized visibility of the target as a function of target and mask durations for 3 observers. Results for different stimulus onset asynchrony (SOA) conditions are with respect to the target-only (T-only) baseline condition, which is normalized to a value of 1 (dotted line). The upper and lower panels correspond to normalized visibilities based on luminance and Michelson contrast values, respectively. Error bars correspond to $1 S E M$. The error bars are not shown for SOA values of $0 \mathrm{msec}$ (upper panel) and $\mathbf{- 2 0}$ msec (lower panel), to avoid clutter. The magnitudes of the error bars for these conditions are similar to those in the other conditions. 
tions changes with exposure duration, the target visibility was normalized according to both luminance values (upper panel) and Michelson contrast (lower panel). Normalization according to Weber contrast yields results similar to luminance-based normalization. The lower panel does not indicate a significant effect of exposure duration on target visibility $[F(5,10)=0.368, p=.619, \varepsilon=0.219]$. Moreover, neither the effect of SOA nor the effect of the interaction between exposure duration and SOA was significant $[F(2,4)=0.197, p=.829 ; F(10,20)=0.898$, $p=.469, \varepsilon=0.170]$. On the other hand, with normalized visibilities based on luminance values (Figure 11, upper panel), there was a slight decrease at the exposure duration of $10 \mathrm{msec}$. However, the effects of exposure duration and SOA also were not significant $[F(5,10)=5.938, p=$ $.087, \varepsilon=0.314 ; F(2,4)=5.321, p=.075]$.

Although the luminance values used in our experiment are much lower than the values in Alpern (1963), our results, shown in Figure 11, are nearly monotonic, like the low inducing bar luminance condition in his study. Our results show that brightness enhancement in the oppositecontrast-polarity condition occurs for small SOA values ranging from 0 to $-20 \mathrm{msec}$. The magnitude of this enhancement appears to be independent of stimulus duration in particular when normalization is made according to Michelson contrast. Therefore, this finding suggests that the enhancement for the opposite-polarity condition and the classical brightness contrast phenomenon may be directly related to each other and stem from the same mechanisms, at least under the stimulus conditions used here.

\section{GENERAL DISCUSSION}

In this study, we investigated paracontrast masking within the context of three putative processes (brief inhibition, facilitation, and prolonged inhibition) first proposed by Breitmeyer et al. (2006). In our previous study (Breitmeyer et al., 2006), these three processes were suggested within the context of the RECOD model (Öğmen, 1993; Öğmen et al., 2003). The model is biologically inspired and extends the dual-channel masking model proposed by Breitmeyer and Ganz (1976). The dual afferent pathways in the model correspond to the retinal ganglion cells with slow-sustained and fast-transient response properties and their parallel projections to the lateral geniculate nucleus (LGN). These two afferent pathways represent the magnodriven (M) transient system and the parvo-driven (P) sustained system. The postretinal layers are lumped representations of the LGN and early parts of the visual cortex. Moreover, the model identifies the postretinal lumped areas that receive dominant $\mathrm{M}$ and $\mathrm{P}$ inputs with transient and sustained channels, respectively. Both target and mask stimuli produce transient and sustained activities in the model. The sustained activity of a mask can reduce the sustained activity of the target, and this is called intrachannel inhibition. Intrachannel inhibition is proposed as the main source for the suppressive mechanisms (brief and prolonged) observed in paracontrast masking. In the model, brief inhibition is produced by a relatively fast intrachannel inhibition realized in the center-surround antagonism of classical receptive fields, and prolonged inhibition is attributed to a slower higher level processing of cortical (postretinal) activity. At this level, activity from an additional subcortical network multiplicatively gates these afferent excitatory P-input signals to the postretinal sustained network. This modulatory effect produces enhancement in the target's visibility (facilitation), which is observed up to a -110-msec SOA value.

We found that the facilitation observed between SOAs of 0 and $-110 \mathrm{msec}$ consistently weakens with an increase in edge-to-edge spatial separation. According to the hypothesis based on lateral excitations propagating at a fixed speed (Polat \& Sagi, 2006; Tanaka \& Sagi, 1998), we would expect a peak shift of approximately $90 \mathrm{msec}$ $\left(0.3^{\circ} \times 300 \mathrm{msec} / \mathrm{deg}\right)$ to more negative SOA values for the $0.3^{\circ} \mathrm{T}-\mathrm{M}$ separation condition. Polat and Sagi (1993, 2006) used Gabor patches in their experiments and found that the increase of the target's visibility is highly dependent on orientation, spatial frequency, and phase difference between the target and flankers. Gabor patches may specifically support the perceptual groupings and completions similar to those found in filling-in boundary gaps and illusory contours. Therefore, these Gabor patches may activate mechanisms based on lateral long-range connections in the visual cortex (Gilbert \& Wiesel, 1989; Grinvald, Lieke, Frostig, \& Hildesheim, 1994), and these connections may increase the detection threshold of the target stimulus. In contrast, our results, obtained by using a disk-ring stimulus configuration, suggest enhancement mechanisms with a fixed delay (as was suggested by Bachmann, 1988, 1994, 1997) but decreasing effects when spatial separation increases.

The results of contrast polarity experiments (Experiments 2 and 3) can be explained by three paracontrast processes. As is depicted in Figure 6, brief inhibition turns into brief facilitation, and this brief facilitation becomes effective at short SOA values in the paracontrast masking function in the opposite-contrast-polarity condition. This effect of contrast polarity on brief inhibition is observed in the surface brightness results (Figure 5). Moreover, the amount of facilitation observed between -20 - and -110 -msec SOA values is highly reduced for the opposite-polarity condition (Figure 5), but prolonged inhibition is not affected by a contrast polarity change (Figures 5 and 10).

The human visual system is considered to be approximately linear in the small neighborhood of the nearthreshold regime, and the impulse response functions in both the time and spatial domains have been investigated (for a review, see Ikeda, 1986). The temporal impulse response of the visual system is generally accepted as either biphasic (Ikeda, 1966; Kelly, 1961) or triphasic (Bowen, 1989; Tyler, 1992). A biphasic temporal impulse response function consists of a positive phase followed by a negative one. Watson and Nachmias (1977) used two subthreshold gratings, presented to the observers with varying SOA values. According to the summation index technique, they found an initial summative or excitatory phase, followed by a weaker subtractive or inhibitory phase. In general, the spatial impulse response is triphasic, with a central posi- 
tive region followed by shallower negative regions on both sides of the central region (King-Smith \& Kulikowski, 1973, 1975; Thomas, 1968). King-Smith and Kulikowski (1975) measured the effect of two parallel (1.2 arc min wide) subthreshold lines on the detectability of a central line of the same width. Their results suggested that the spatial response profile is characterized by a central summative or excitatory region flanked by two symmetrical subtractive or inhibitory regions.

The results in Figure 5 can also be explained by using the spatiotemporal impulse response function of the visual system as follows. In the same-polarity condition, the surrounding mask initially reduces the visibility of the target, and it starts increasing the target's visibility when it changes its temporal phase. On the other hand, the opposite-polarity mask first enhances and then gradually starts inhibiting the target's visibility as the mask changes its temporal phase. However, exactly how and where in the visual system the target and mask spatiotemporal responses interact at suprathreshold level remains to be answered. Manahilov (1995) used two narrow suprathreshold bars to examine the spatiotemporal response of the visual system. The inducing stimulus (IS) was a 30-cd $/ \mathrm{m}^{2}$ decrement below the mean luminance, and the test stimulus (TS) was a $45-\mathrm{cd} / \mathrm{m}^{2}$ increment above the mean luminance. The stimulus duration was $15 \mathrm{msec}$. In order to elicit the temporal response to the IS, the perceived brightness of the TS was measured for different SOAs and center-to-center separations between the IS and TS. When the spatial separation was zero - that is, when the two stimuli overlapped - the temporal response to the IS had three phases: a large central inhibitory phase and two temporally flanking excitatory phases. The temporal response function was almost symmetric and reached its peak facilitation at approximately 50-msec SOA for both metacontrast and paracontrast. Then the center-to-center separation between the IS and TS was varied for SOAs of $-50,0$, and $50 \mathrm{msec}$. The spatial spread of the response depended strongly on SOA value, and almost no effect was observed when the edge-to-edge distance became greater than zero. Since the target and mask did not overlap in our experiments, it is hard to compare our results (especially those for the opposite-polarity condition in Figure 5) with those in this study.

When we compare the surface brightness results with contour identification judgment results, it is obvious that the two different tasks yield distinct paracontrast masking functions, replicating the results in Breitmeyer et al. (2006). Moreover, the masking function in the contour identification task is less sensitive to contrast polarity change than is that in the surface brightness task at SOA values smaller than $-20 \mathrm{msec}$. Effects of contrast polarity in metacontrast have also been reported by Breitmeyer (1978) and Becker and Anstis (2004). Both studies used disk and ring as target and mask stimuli, respectively. Breitmeyer adopted the contour identification task and obtained U-shaped metacontrast masking functions for both same- and opposite-polarity conditions. On the other hand, Becker and Anstis used the perceived surface brightness of the target disk to index the magnitude of metacontrast suppression. On the basis of the single, relatively large SOA value $(133 \mathrm{msec})$ used in their experiment, their results did not indicate any significant suppression when the target and mask had opposite contrast polarity. Recently, Breitmeyer et al. (2008) investigated the effect of contrast polarity in metacontrast by using brightness judgments for a broad range of SOA values. The results showed that substantial metacontrast suppression can be observed in the opposite-contrast-polarity condition around the SOA value of $60 \mathrm{msec}$. Thus, the failure to observe metacontrast suppression for the opposite-contrast-polarity condition in Becker and Anstis's study stemmed from their use of a single, relatively large SOA value. As in metacontrast studies, our results in this study show that the effect of contrast polarity on paracontrast depends strongly on the value of SOA.

In this study, we investigated the dependence of paracontrast masking on contrast polarity and $\mathrm{T}-\mathrm{M}$ separation. In our previous study (Breitmeyer et al., 2006), we investigated the effect of $\mathrm{M} / \mathrm{T}$ contrast ratio on paracontrast by using a wider range of mask luminance values $\left(0.5,30.5,56 \mathrm{~cd} / \mathrm{m}^{2}\right.$, with the background luminance set at $95 \mathrm{~cd} / \mathrm{m}^{2}$ ). Our results showed that, for surface brightness judgments, the facilitation increases as the $\mathrm{M} / \mathrm{T}$ contrast ratio increases. The dip around $\mathrm{SOA}=-10 \mathrm{msec}$ becomes more pronounced as the $\mathrm{M} / \mathrm{T}$ contrast ratio decreases. These findings can be explained by our model if we assume that an increase in mask contrast causes a stronger enhancement of facilitation, as compared with brief inhibition. For contour judgment, we found the brief inhibition to increase with increasing $\mathrm{M} / \mathrm{T}$ contrast ratio. As was mentioned before, in the case of contour processes, we postulate the facilitatory process to be weak. Thus, the increase in mask contrast appears to be a net enhancement of the inhibitory process, relative to the facilitatory process. Further parametric and modeling studies may test these suggestions and shed more light on how the underlying mechanism of facilitation interacts with the suppressive mechanisms during paracontrast.

\section{AUTHOR NOTE}

This work was supported by NSF Grant BCS-0114533 and NIH Grant R01-MH49892. Correspondence concerning this article should be addressed to H. Kafaligönül, Vision Center Laboratory, Salk Institute for Biological Studies, 10010 N. Torrey Pines Rd., La Jolla, CA 92037 (e-mail: hulusi.kafaligonul@gmail.com).

\section{REFERENCES}

ALPERN, M. (1963). Simultaneous brightness contrast for flashes of light of different durations. Investigative Ophthalmology \& Visual Science, 2, 47-54

BACHMANN, T. (1988). Time course of the subjective contrast enhancement for a second stimulus in successively paired above-threshold transient forms: Perceptual retouch instead of forward masking. $\mathrm{Vi}$ sion Research, 28, 1255-1261.

Bachmann, T. (1994). Psychophysiology of visual masking: The fine structure of conscious experience. Commack, NY: Nova.

BaCHMANN, T. (1997). Visibility of brief images: The dual-process approach. Consciousness \& Cognition, 6, 491-518.

BECKER, M. W., \& ANSTIS, S. (2004). Metacontrast masking is specific to luminance polarity. Vision Research, 44, 2537-2543.

Benardete, E. A., \& Kaplan, E. (1997). The receptive field of the 
primate P retinal ganglion cell: I. Linear dynamics. Visual Neuroscience, 14, 169-185.

Berman, N. J., Douglas, R. J., Martin, K. A., \& Whitteridge, D. (1991). Mechanisms of inhibition in cat visual cortex. Journal of Physiology, 440, 697-722.

Bowen, R. W. (1989). Two pulses seen as three flashes: A superposition analysis. Vision Research, 29, 409-417.

BreitMeyer, B. G. (1978). Metacontrast with black and white stimuli: Evidence of inhibition of on- and off-sustained activity by either onor off-transient activity. Vision Research, 18, 1443-1448.

BreitMeyer, B. G., \& GANZ, L. (1976). Implications of sustained and transient channels for theories of visual pattern masking, saccadic suppression and information processing. Psychological Review, 83, 1-36.

Breitmeyer, B. G., Kafaligönül, H., ÖĞMen, H., Mardon, L., TODD, S., \& Ziegler, R. (2006). Meta- and paracontrast reveal differences between contour- and brightness-processing mechanisms. Vision Research, 46, 2645-2658.

Breitmeyer, B. G., \& ÖĞMEN, H. (2000). Recent models and findings in visual backward masking: A comparison, review, and update. Perception \& Psychophysics, 62, 1572-1595.

Breitmeyer, B. G., \& ÖĞMEn, H. (2006). Visual masking: Time slices through conscious and unconscious vision ( 2 nd ed.). Oxford: Oxford University Press.

BreitMeyer, B. G., ÖĞMEN, H., \& Chen, J. (2004). Unconscious priming by color and form: Different processes and levels. Consciousness \& Cognition, 13, 138-157.

Breitmeyer, B. G., ÖĞMEn, H., Ramon, J., \& Chen, J. (2005). Unconscious priming by form and their parts. Visual Cognition, 12, 720-736.

Breitmeyer, B. G., TaPia, E., KafaligönÜl, H., \& ÖĞMEN, H. (2008). Metacontrast masking and stimulus contrast polarity. Vision Research, 48, 2433-2438.

DRESP, B., \& BonNeT, C. (1995). Subthreshold summation with illusory contours. Vision Research, 35, 1071-1078.

Foley, J. M., \& ChEN, C.-C. (1999). Pattern detection in the presence of maskers that differ in spatial phase and temporal offset: Threshold measurements and a model. Vision Research, 39, 3855-3872.

Francis, G. (1997). Cortical dynamics of lateral inhibition: Metacontrast masking. Psychological Review, 104, 572-594.

Georgeson, M. A. (1988). Spatial phase dependence and the role of motion detection in monocular and dichoptic forward masking. Vision Research, 28, 1193-1205.

Gilbert, C. D., \& Wiesel, T. N. (1989). Columnar specificity of intrinsic horizontal and corticocortical connections in cat visual cortex. Journal of Neuroscience, 9, 2432-2442.

Grinvald, A., Lieke, E. E., Frostig, R. D., \& Hildesheim, R. (1994). Cortical point-spread function and long-range lateral interactions revealed by real-time optical imaging of macaque monkey primary visual cortex. Journal of Neuroscience, 14, 2545-2568.

Grossberg, S. (1994). 3-D vision and figure-ground separation by visual cortex. Perception \& Psychophysics, 55, 48-120.

GrossberG, S. (2003). Filling-in the forms: Surface and boundary interactions in visual cortex. In L. Pessoa \& P. De Weerd (Eds.), Filling-in: From perceptual completion to cortical reorganization (pp. 13-37). Oxford: Oxford University Press.

Heinemann, E. G. (1955). Simultaneous brightness induction as a function of inducing- and test-field luminances. Journal of Experimental Psychology, 50, 89-96.

Hermens, F., Luksys, G., Gerstner, W., Herzog, M. H., \& Ernst, U. (2008). Modeling spatial and temporal aspects of visual backward masking. Psychological Review, 115, 83-100.

IKEDA, M. (1966). Temporal summation of positive and negative flashes in the visual system. Journal of the Optical Society of America, 55, $1527-1534$

IKEDA, M. (1986). Temporal impulse response. Vision Research, 26, $1431-1440$

Kelly, D. H. (1961). Visual responses to time-dependent stimuli: II. Single-channel model of the photopic visual system. Journal of the Optical Society of America, 51, 747-757.
King-Smith, P. E., \& KULIKowsKi, J. J. (1973). Spatial arrangement of the flicker and pattern detectors for a fine line. Journal of Physiology, 234, 33P-35P.

King-Smith, P. E., \& Kulikowski, J. J. (1975). Pattern and flicker detection analysed by subthreshold summation. Journal of Physiology, 249, 519-548

Maffei, L., Cervetto, L., \& Fiorentini, A. (1970). Transfer characteristics of excitation and inhibition in cat retinal ganglion cells. Journal of Neurophysiology, 33, 276-284.

Manahilov, V. (1995). Spatiotemporal visual response to suprathreshold stimuli. Vision Research, 35, 227-237.

Nelson, S. B. (1991). Temporal interactions in the cat visual system: I. Orientation-selective suppression in the visual cortex. Journal of Neuroscience, 11, 344-356.

ÖĞMEN, H. (1993). A neural theory of retino-cortical dynamics. Neural Networks, 6, 245-273.

ÖĞMen, H., Breitmeyer, B. G., \& Melvin, R. (2003). The what and where in visual masking. Vision Research, 43, 1337-1350.

Otto, T. U., ÖĞMen, H., \& Herzog, M. H. (in press). Feature integration across space, time, and orientation. Journal of Experimental Psychology: Human Perception \& Performance.

Peterhans, E., \& von Der Heydt, R. (1989). Mechanisms of contour perception in monkey visual cortex: II. Contours bridging gaps. Journal of Neuroscience, 9, 1749-1763.

Poggio, G. B., Baker, F. H., Lamarre, Y., \& Sanseverino, E. R. (1969). Afferent inhibition at input to visual cortex of the cat. Journal of Neurophysiology, 32, 892-915.

Polat, U., \& SAGI, D. (1993). Lateral interactions between spatial channels: Suppression and facilitation revealed by lateral masking experiments. Vision Research, 33, 993-999.

Polat, U., \& SAGI, D. (2006). Temporal asymmetry of collinear interactions. Vision Research, 46, 953-960.

Polat, U., Sterkin, A., \& Yehezkel, O. (2007). Spatio-temporal lowlevel neural networks account for visual masking. Advances in Cognitive Psychology, 3, 153-165.

SAYim, B., Westheimer, G., \& Herzog, M. H. (2008). Contrast polarity, chromaticity, and stereoscopic depth modulate contextual interactions in vernier acuity. Journal of Vision, 8, Art. 12, 1-9.

Singer, W., \& Creutzfeldt, O. D. (1970). Reciprocal lateral inhibition of on- and off-center neurones in the lateral geniculate body of the cat. Experimental Brain Research, 10, 311-330.

TANAKA, Y., \& SAGI, D. (1998). Long-lasting, long-range detection facilitation. Vision Research, 38, 2591-2599.

Thomas, J. P. (1968). Linearity of spatial integrations involving inhibitory interactions. Vision Research, 8, 49-60.

Tyler, C. W. (1992). Psychophysical derivation of the impulse response through generation of ultrabrief responses: Complex inverse estimation without minimum-phase assumptions. Journal of the Optical Society of America A, 9, 1025-1040.

Ursino, M., \& LA CARA, G.-E. (2004a). Comparison of different models of orientation selectivity based on distinct intracortical inhibition rules. Vision Research, 44, 1641-1658.

Ursino, M., \& La CARA, G.-E. (2004b). A model of contextual interactions and contour detection in the primary visual cortex. Neural Networks, 17, 719-735.

Watson, A. B., \& NaCHMiAs, J. (1977). Patterns of temporal interaction in the detection of gratings. Vision Research, 17, 893-902.

XING, J., \& HeEgER, D. J. (2000). Center-surround interactions in foveal and peripheral vision. Vision Research, 40, 3065-3072.

Yen, S.-C., \& FinKel, L. H. (1998). Extraction of perceptually salient contours by striate cortical networks. Vision Research, 38, 719-741.

Zhou, H., Friedman, H. S., \& von der Heydt, R. (2000). Coding of border ownership in monkey visual cortex. Journal of Neuroscience, 20, 6594-6611.

(Manuscript received March 25, 2008; revision accepted for publication April 13, 2009.) 